\title{
Personalization of Repetitive Transcranial Magnetic Stimulation for the Treatment of Major Depressive Disorder According to the Existing Psychiatric Comorbidity
}

\author{
Po-Han Chou ${ }^{1,2,3,4, *}$, Yen-Feng Lin ${ }^{4,5,6,7, *}$, Ming-Kuei Lu $^{8,9}$, Hsin-An Chang ${ }^{10}$, Che-Sheng Chu ${ }^{11,12,13}$, \\ Wei Hung Chang ${ }^{14,15}$, Taishiro Kishimoto ${ }^{16}$, Alexander T. Sack ${ }^{17,18}$, Kuan-Pin Su ${ }^{2,19,20,21}$ \\ ${ }^{1}$ Department of Psychiatry, China Medical University Hsinchu Hospital, China Medical University, Hsinchu, ${ }^{2}$ Department of Psychiatry, China \\ Medical University Hospital, China Medical University, Taichung, ${ }^{3}$ Department of Biological Science and Technology, National Chiao Tung \\ University, Hsinchu, ${ }^{4}$ Taiwan Allied Clinics for Integrative TMS, Taipei, ${ }^{5}$ Center for Neuropsychiatric Research, National Health Research Institutes, \\ Miaoli, ${ }^{6}$ Department of Public Health \& Medical Humanities, Faculty of Medicine, National Yang-Ming Chiao Tung University, Taipei, 'Balance \\ Psychiatric Clinic, Hsinchu, ${ }^{8} \mathrm{Ph}$.D. Program for Translational Medicine, College of Medicine, China Medical University, ${ }^{9}$ Department of Neurology, \\ China Medical University Hospital, Taichung, ${ }^{10}$ Department of Psychiatry, Tri-Service General Hospital, National Defense Medical Center, Taipei, \\ ${ }^{11}$ Department of Psychiatry, Kaohsiung Veterans General Hospital, ${ }^{12}$ Center for Geriatric and Gerontology, Kaohsiung Veterans General Hospital, \\ ${ }^{13}$ Graduate Institute of Medicine, College of Medicine, Kaohsiung Medical University, Kaohsiung, ${ }^{14}$ Department of Psychiatry, National Cheng \\ Kung University Hospital, College of Medicine, National Cheng Kung University, Tainan, ${ }^{15}$ Department of Psychiatry, National Cheng Kung \\ University Hospital, Dou-Liou Branch, Yunlin, Taiwan, ${ }^{16}$ Department of Neuropsychiatry, Keio University School of Medicine, Tokyo, Japan, \\ ${ }^{17}$ Department of Cognitive Neuroscience, Faculty of Psychology and Neuroscience, Maastricht University, ${ }^{18}$ Department of Psychiatry and \\ Neuropsychology, School for Mental Health and Neuroscience (MHeNs), Brain + Nerve Centre, Maastricht University Medical Centre+ (MUMC \\ + ), Maastricht, The Netherlands, ${ }^{19}$ College of Medicine, China Medical University, ${ }^{20}$ Mind-Body Interface Laboratory (MBI-Lab), China Medical \\ University Hospital, Taichung, ${ }^{21}$ An-Nan Hospital, China Medical University, Tainan, Taiwan
}

Repetitive transcranial magnetic stimulation (rTMS) and intermittent theta-burst stimulation (iTBS) are evidenced-based treatments for patients with major depressive disorder (MDD) who fail to respond to standard first-line therapies. However, although various TMS protocols have been proven to be clinically effective, the response rate varies across clinical applications due to the heterogeneity of real-world psychiatric comorbidities, such as generalized anxiety disorder, posttraumatic stress disorder, panic disorder, or substance use disorder, which are often observed in patients with MDD. Therefore, individualized treatment approaches are important to increase treatment response by assigning a given patient to the most optimal TMS treatment protocol based on his or her individual profile. This literature review summarizes different rTMS or TBS protocols that have been applied in researches investigating MDD patients with certain psychiatric comorbidities and discusses biomarkers that may be used to predict rTMS treatment response. Furthermore, we highlight the need for the validation of neuroimaging and electrophysiological biomarkers associated with rTMS treatment responses. Finally, we discuss on which directions future efforts should focus for developing the personalization of the treatment of depression with rTMS or iTBS.

KEY WORDS: Depression; Brain stimulation; Repetitive transcranial magnetic stimulation; rTMS; Theta-burst stimulation; TBS.

\section{INTRODUCTION}

Major depressive disorder (MDD) is a leading cause of

Received: December 14, 2020 / Accepted: December 24, 2020 Address for correspondence: Kuan-Pin Su

Department of Psychiatry, China Medical University Hospital, No. 2, Yuh-Der Road, Taichung 404, Taiwan

E-mail: cobolsu@gmail.com

ORCID: https://orcid.org/0000-0002-4501-2502

*These authors contributed equally to this study as co-first authors. disability worldwide and is a major contributor to the global burden of disease. Although antidepressants are clinically effective, many unmet clinical needs such as insufficiency of efficacy and presence of side effects urge the development of novel therapeutic strategies. Repetitive transcranial magnetic stimulation (rTMS), a novel therapeutic strategy, has been approved for the treatment of MDD by the U.S. Food and Drug Administration (FDA)

(c) This is an Open-Access article distributed under the terms of the Creative Commons Attribution Non-Commercial License (http://creativecommons.org/licenses/by-nc/4.0) which permits unrestricted non-commercial use, distribution, and reproduction in any medium, provided the original work is properly cited. 
and is considered as the first-line treatment in many countries [1,2]. The left dorsolateral prefrontal cortex (DLPFC) has been found to be hypoactive in depression [3], and high-frequency (HF)-rTMS over this region has been studied most intensively and applied clinically. Two multicenter randomized control trials (RCTs) on rTMS for the treatment of MDD were conducted with high methodological quality [4,5], and their positive results played a major role in the approval of rTMS for the treatment of treatment-resistant depression (TRD). Both studies used a protocol of HF-rTMS of the left DLPFC, performed at $120 \%$ of the resting motor threshold (RMT). A train consists of 40 pulses $(10 \mathrm{~Hz}$ for a total of 4 seconds), and a total of 75 trains (i.e., 3,000 pulses) were used. In addition, the antidepressant effects of HF-rTMS of the left DLPFC have been confirmed in many other RCTs and recent meta-analyses, and thus HF-rTMS of the left DLPFC has been considered as the standard rTMS protocol with the highest level of efficacy for the treatment of depression $[6,7]$. However, rates of response to HF-rTMS vary among patients with MDD, probably because of the differences in the presence of other frequently occurring psychiatric comorbidities, such as anxiety disorders [8]. Therefore, we aimed to review the efficacies of different rTMS protocols in patients with MDD patients showing other psychiatric comorbidities and to recommend personalized treatment protocols for clinical practice. Moreover, we aimed to discuss patient factors and neuroimaging or neurophysiological biomarkers that could be used as potential biomarkers of rTMS treatment response.

\section{OTHER rTMS PROTOCOLS FOR THE TREATMENT OF DEPRESSION}

rTMS protocols for the treatment of depression are generally based on the prefrontal asymmetry theory [9], i.e., relative hypoactivity of the left DLPFC and relative hyperactivity of the right DLPFC in depression. rTMS is known to change brain electrical activity [10]. Low-frequency $(\mathrm{LF} ; \leq 1 \mathrm{~Hz}$ )-rTMS or continuous theta-burst stimulation (cTBS) has an inhibitory effect, whereas HF-rTMS ( $\geq 5$ $\mathrm{Hz}$ ) or intermittent theta-burst stimulation (iTBS) produces excitatory effects on the neuron. According to the hypothesis of asymmetric prefrontal activity in depression, it was believed that both excitatory rTMS (HF or iTBS) of the left and inhibitory rTMS (LF or CTBS) of the right DLPFC can be effective in treating MDD [11]. On the basis of two previous review articles $[6,12]$, commonly used rTMS protocols in previous studies are shown in Table 1.

In addition to HF- or LF-unilateral rTMS, "bilateral" rTMS, which is performed both as sequential LF-rTMS of the right DLPFC and HF-rTMS of the left DLPFC in the same session, has been studied and applied clinically [13]. These interventions have been reported to be more effective than a sham intervention on improving depressive symptoms [14], although the effect size is modest. Regarding bilateral TBS (biTBS), the first randomized sham-controlled trial comparing antidepressant efficacy among different kinds of TBS protocols reported that biTBS (i.e., iTBS to the left and cTBS to the right DLPFC) had the best results than the other protocols [15]. A recent RCT also supports the efficacy and tolerability of biTBS monotherapy for patients with MDD [16].

Several previous studies found no difference in the efficacy of LF-rTMS of the right DLPFC and HF-rTMS of the

Table 1. Common TMS parameters used in previous researches

\begin{tabular}{lllr}
\hline \multicolumn{1}{c}{ Frequency } & \multicolumn{1}{c}{ Intensity } & \multicolumn{1}{c}{ Target } & Number of pulses/session \\
\hline $10 \mathrm{~Hz} \mathrm{rTMS}$ & $80-120 \%$ RMT & Left DLPFC & $1,200-3,000$ \\
$1 \mathrm{~Hz} \mathrm{rTMS}$ & $80-120 \%$ RMT & Right DLPFC & $120-1,200$ \\
iTBS & $80-120 \% \mathrm{MT}$ & Left DLPFC & $600-3,600$ \\
cTBS & $80-90 \% \mathrm{MT}$ & Right DLPFC & $600-3,600$ \\
FDA approved TMS protocol & $120 \%$ RMT & Left DLPFC \\
10 Hz & & & 3,000 \\
FDA approved TBS protocol & $120 \%$ RMT & Left DLPFC \\
iTBS
\end{tabular}

TMS, transcranial magnetic stimulation; rTMS, repetitive TMS; TBS, theta-burst stimulation; iTBS, intermittent TBS; cTBS, continuous TBS; RMT, resting motor threshold; MT, motor threshold; DLPFC, dorsolateral prefrontal cortex; FDA, U.S. Food and Drug Administration. 
left DLPFC for the treatment of MDD. A meta-analysis that included twelve RCTs with 175 and 186 patients with MDD undergoing HF-rTMS of the left DLPFC and LF-rTMS of the right DLPFC, respectively, found no difference in the treatment response between the two protocols [17]. In this meta-analysis, the mean number of treatment sessions was 14.6 with a mean total rTMS pulse number of 19,708 for HF-rTMS and 9,425 for LF-rTMS, and the reported response rates were $44.6 \%$ and $40.9 \%$, respectively (odds ratio $[\mathrm{OR}], 1.08 ; 95 \%$ confidence interval $[\mathrm{Cl}], 0.88-$ 1.34). Furthermore, remission rates were compared in five studies that included a total of 131 patients with MDD, and these studies found no statistically significant difference (64 patients received HF-rTMS of the left DLPFC and 67 patients received LF-rTMS of the right DLPFC; OR, 1.29; 95\% Cl, 0.54-3.10) [17]. A network meta-analysis (NMA) that included 81 RCTs with 4,233 patients found that priming LF-rTMS (OR, 4.66; 95\% Cl, 1.70-12.77), bilateral rTMS (OR, 3.96; 95\% Cl, 2.37 -6.60), HF-rTMS $(\mathrm{OR}, 3.07 ; 95 \% \mathrm{Cl}, 2.24-4.21)$, TBS (OR, 2.54; $95 \% \mathrm{Cl}$, $1.07-6.05)$, and LF-rTMS (OR, 2.37; 95\% Cl, $1.52-$ 3.68) were more effective than sham intervention and that all interventions were at least as acceptable as sham intervention [14]. The estimated relative ranking of treatments implied that priming LF-rTMS and bilateral rTMS might show the best performance among all rTMS interventions in terms of efficacy and tolerability. However, the body of evidence supporting this statement is small with varied quality; results of such meta-analyses should be interpreted very cautiously $[18,19]$.

European and Canadian experts have recommended the use of HF- and LF-rTMS as first-line protocols for the treatment of depression $[1,6]$. In addition, several authors pointed out that HF-rTMS might have a greater potential to accelerate and improve the clinical response to antidepressants than LF-rTMS, whereas LF-rTMS might have a better tolerability profile than HF-rTMS in patients with agitation [20]. In summary, current evidence suggests that HF-rTMS, LF-rTMS, and bilateral rTMS may be equivalently effective in the treatment of MDD; larger RCTs should be performed to directly compare the treatment efficacy and acceptability among these protocols.

\section{rTMS PROTOCOLS FOR THE TREATMENT OF DEPRESSION WITH COMORBID PSYCHIATRIC CONDITIONS}

rTMS has been considered as treatment option for patients with comorbid MDD and other psychiatric disorders, especially for those who did not show adequate response to antidepressant medications. MDD is frequently associated with other psychiatric comorbidities, which leads to an increase in the severity of symptoms and worsening of treatment response and prognosis [21]. The STAR*D trial found that adults patients with MDD showed high prevalence rates of current social anxiety disorder (SAD: 25.6\%), generalized anxiety disorder (GAD: $20.8 \%$ ), posttraumatic stress disorder (PTSD: 18.8\%), obsessive-compulsive disorder (OCD: $13.4 \%$ ), and panic disorder (PD: 11.1\%) [22]. Although the issue of comorbidities has been recognized, few studies to date have evaluated the efficacy of rTMS for the treatment of patients with MDD showing other psychiatric comorbidities and provided specific recommendations for the treatment of

Table 2. Potential rTMS treatment protocols for MDD with and without comorbid psychiatric disorders

\begin{tabular}{|c|c|c|}
\hline Diagnosis & Potential rTMS protocols & Rationale \\
\hline $\begin{array}{l}\text { MDD (including } \\
\text { anxious depression) }\end{array}$ & $\begin{array}{l}\text { Left sided HF-rTMS; } \\
\text { Right sided LF-rTMS; } \\
\text { Bilateral rTMS }\end{array}$ & Prefrontal asymmetry theory in depression \\
\hline $\begin{array}{l}\text { MDD + Anxiety } \\
\text { disorders }\end{array}$ & $\begin{array}{l}\text { Right sided LF-rTMS; } \\
\text { Bilateral rTMS }\end{array}$ & Hyperactive right DLPFC in anxiety \\
\hline MDD + PTSD & $\begin{array}{l}\text { Insufficient evidence to make recommendations; } \\
\text { Left sided 5-Hz rTMS, right sided HF-rTMS, and right } \\
\text { sided iTBS warrant further researches }\end{array}$ & $\begin{array}{l}\text { Abnormal frontal theta }(4-7 \mathrm{~Hz}) \text { activity in PTSD } \\
\text { Associations between hypoactive HPA axis and right } \\
\text { hypofrontality }\end{array}$ \\
\hline $\begin{array}{l}\text { MDD + Substance } \\
\text { use disorders }\end{array}$ & $\begin{array}{l}\text { Insufficient evidence to make recommendations; } \\
\text { Left sided HF-rTMS warrants further researches }\end{array}$ & $\begin{array}{l}\text { Hypoactive left DLPFC in patients with substance } \\
\text { dependence }\end{array}$ \\
\hline
\end{tabular}

rTMS, repetitive transcranial magnetic stimulation; MDD, major depressive disorder; PTSD, posttraumatic stress disorder; HF, high frequency; LF, low frequency; iTBS, intermittent theta-burst stimulation; DLPFC, dorsolateral prefrontal cortex; HPA, hypothalamic-pituitary-adrenal. 
these complex clinical situations using this therapeutic strategy. In this section, we summarized the evidence on the effectiveness of rTMS for the treatment of MDD with comorbid anxiety disorders and substance use disorders (Table 2).

\section{rTMS Protocols for MDD with Comorbid Anxiety Disorders}

Some current evidence suggests that right-sided LF-rTMS or bilateral rTMS may be effective to improve both depressive and anxiety symptoms in MDD with comorbid anxiety disorders. According to a literature review, rTMS studies for treating anxiety disorders were few, and LF-rTMS (1 Hz) of the right DLPFC has been considered as a treatment option for the treatment of anxiety disorders [23]. Previous neurophysiological and imaging studies suggest that increased right DLPFC activity may be related to an increase in anxiety [24]. In addition to considering the prefrontal asymmetry theory of depression, it is reasonable to use right-sided LF-rTMS or bilateral rTMS as the potential treatment protocol for MDD with comorbid anxiety disorders. In a small pilot study [25], thirteen adult patients with comorbid MDD and GAD received sequential bilateral treatments, with LF-rTMS (1,000 pulses at 1 $\mathrm{Hz}$ frequency) of the right DLPFC followed by HF-rTMS $(10 \mathrm{~Hz})$ of the left DLPFC. The number of treatment sessions ranged from 24 to 36 over a period of 5-6 weeks. At the end of the treatment period, 10 out of 13 patients (76.9\%) achieved remission of depression, with a score of $<8$ on the 21 -item Hamilton Rating Scale for Depression (HAMD-21), and 11 out of 13 patients (84.6\%) achieved remission of anxiety symptoms with a GAD-7 score $<5$. Overall, after the bilateral rTMS treatment, decreases of $65 \%$ and $75 \%$ in depression and anxiety scores, respectively, were observed [25]. Another naturalistic clinical study [26] examined the effectiveness of rTMS for the treatment of treatment-resistant MDD with or without comorbid anxiety disorders (GAD, 102 patients; OCD, 32 patients; PTSD, 26 patients; PD with agoraphobia, 41 patients; agoraphobia, 61 patients; SAD, 58 patients; and no anxiety disorders, 72 patients). Patients were treated with either sequential bilateral or right LF-rTMS at $110 \%$ of the RMT. The sequential bilateral protocol involved $15 \mathrm{mi}-$ nutes of $10 \mathrm{~Hz}$ rTMS, delivered in 5-second trains with a 25 seconds intertrain interval (1,500 pulses), to the left DLPFC, followed by 15 minutes of $1 \mathrm{~Hz}$ rTMS (900 pulses) to the right DLPFC. The unilateral right protocol involved 15 minutes of $1 \mathrm{~Hz}$ rTMS (900 pulses) to the right DLPFC. rTMS was administered 3 days/week for 6 weeks (18 treatment session in total) or 5 days/week for 4 weeks (20 treatment session in total). After treatment with either the right sided LF or bilateral rTMS, improvement in depressive symptoms was observed in both patients with and without comorbid anxiety disorders, with no significant difference in remission rates between groups. In patients with comorbid anxiety disorders, 39.5\% met response criteria and $23.3 \%$ met criteria for remission. In addition, the researchers found no significant difference between the groups with respect to response rate $(p<0.795)$. Both groups showed significant reductions in HAMD-21, the Montgomery-Asberg Depression Scale, the Zung Self-Rating Depression Scale, and the Hamilton Anxiety Rating Scale scores ( $p<0.001$ for all). Another randomized shamcontrolled trial on the efficacy of rTMS for the treatment of PD with comorbid MDD [27] revealed significantly better improvement in panic symptoms after the administration of LF-rTMS to the right DLPFC ( $1 \mathrm{~Hz}$ frequency; 1,800 pulses/day, once a day, 5 days a week, at $110 \%$ of the RMT) than after that of sham rTMS at week 4 and significant improvement in both panic and depressive symptoms at week 8. In conclusion, regarding the use of rTMS in patients with comorbid MDD and anxiety disorders, although evidence is limited, right-sided LF-rTMS, or bilateral rTMS may be effective in reducing symptoms of both anxiety and depression.

\section{rTMS Protocols for Anxious Depression}

Approximately one-half of patients with MDD experience clinically meaningful levels of anxiety, and these patients may show very severe depressive symptoms, long duration of current episode, increased suicidal ideation, and high number of medical comorbidities and are less likely to respond to antidepressant treatment $[28,29]$. The Diagnostic and Statistical Manual of Mental Disorders 5th edition added "anxious distress" as a category specifier to the diagnosis of MDD for identifying those patients whose anxiety must be considered in their treatment plan. The symptoms listed for anxious distress include tenseness, restlessness, difficulty in concentrating because of worry, fear that something awful may happen, and feelings of loss of control.

rTMS protocols for the treatment of depression, includ- 
ing HF-rTMS, LF-rTMS, and bilateral rTMS, appear to be effective in reducing both depression and anxiety in anxious depression. In a clinical study [30], 32 patients with TRD, including eight with anxious depression, received HF-rTMS to the left DLPFC at $80-130 \%$ of the RMT $(3,000-5,000$ pulses/session) for an average of 31 treatment sessions (standard deviation $=5.39$, range $=22-$ 42). From baseline to the end of treatment, symptoms of both depression and anxiety improved significantly. Patients with and without anxious depression demonstrated similar levels of improvement in depression, and those with anxious depression demonstrated a greater improvement in anxiety [30]. A pooled analysis of data from three clinical trials compared the relative efficacies of three commonly used rTMS protocols for the treatment of anxiety symptoms in patients with depression [31]. A total of 697 patients with TRD and anxiety symptoms underwent a 4-week course of left-sided HF-rTMS ( $\mathrm{n}=179)$, right-sided LF-rTMS ( $n=218$ ), or sequential bilateral rTMS $(n=300)$. All three protocols were similarly effective in treating the symptoms of anxiety and depression, suggesting therapeutic equivalence across the three protocols. Improvement in the severity of depressive symptoms was positively correlated with the improvement in anxiety. Both patients with high and low baseline anxiety scores showed overall symptom reduction [31]. In summary, HF-rTMS, LF-rTMS, and bilateral rTMS showed similar significant treatment effects in patients with MDD with and without anxiety symptoms although those with anxious depression were previously reported to show poor response to antidepressant medications.

\section{rTMS Protocols for Comorbid MDD and PTSD}

Approximately one-half of patients with PTSD are diagnosed of MDD [32,33], and these patients were reported to demonstrate a poorer response to antidepressant treatment $[34,35]$ and a more chronic course of functional impairment [36] than are those with PTSD or MDD alone. Previous studies have provided some evidence supporting the therapeutic effect of rTMS on PTSD [37]. Furthermore, a recent study [38] found that comorbid PTSD did not impact the outcome of rTMS for MDD in a sample of veterans. Therefore, it is of interest to evaluate the efficacy of rTMS for the treatment of MDD with comorbid PTSD and the benefit of its clinical use.

Although evidence is limited, $5 \mathrm{~Hz}$ rTMS to the left
DLPFC and $1 \mathrm{~Hz}$ rTMS to the right DLPFC have shown potential for treatment of comorbid MDD and PTSD. An early small open-label study suggests that left-sided rTMS with a frequency of either $1 \mathrm{~Hz}$ or $5 \mathrm{~Hz}$ showed clinically significant antidepressant effect but only minimal improvement in PTSD symptoms in patients with comorbid MDD and PTSD [39]. In a double-blind, cross-over, sham-controlled study, twenty 30-minutes sessions of LF-rTMS (1 $\mathrm{Hz}$ ) of the right DLPFC showed a larger effect size of improvement in hyperarousal symptoms than did sham intervention [40]. A retrospective chart-review study [41] and a prospective, open-label trial [42] conducted by the same group found that $5 \mathrm{~Hz}$ rTMS of the left DLPFC could significantly reduce symptoms of both PTSD and MDD. In the latter trial, among 35 patients who received up to 40 treatment sessions with 3,000-4,000 pulses/session at 120\% RMT, 23 participants (48.6\%) met a pre-defined PTSD response criterion, 15 (42.9\%) showed response, and $12(34.3 \%)$ showed remission of MDD [42]. Excessive frontal theta activity and abnormal beta activity modulation by the former were reported to be related to the intensity of negative emotional experience in PTSD [43]; thus, the $5 \mathrm{~Hz}$ frequency, that approximates theta band signals $(4-7 \mathrm{~Hz})$ on electroencephalography (EEG), has been studied for its potential to modulate abnormal brain activity in PTSD [41].

Several RCTs have suggested right-sided HF-rTMS $[44,45]$ and iTBS [46] to be possible effective treatment options for PTSD. In these RCTs, although participants were not necessarily having comorbid MDD, depressive symptoms were highly prevalent and measured as an index of improvement after treatments. It is possible that conventional HF-rTMS protocol or iTBS protocol is also effective for the treatment of patients with comorbid MDD and PTSD. Previous animal studies suggested an association between right hypofrontality and hypoactivity of the hypothalamic-pituitary-adrenal (HPA) axis in PTSD; therefore, the improvement in PTSD symptoms with right-sided HF-rTMS is possibly the result of activation of the HPA axis [45].

According to current evidence, it is difficult to recommend a specific rTMS protocol for patients with comorbid MDD and PTSD. Further well-designed RCTs would be needed to evaluate the efficacies of different treatment protocols and to validate the effectiveness of $5 \mathrm{~Hz}$ rTMS and the right-sided excitatory rTMS. 


\section{rTMS Protocols for Comorbid MDD and Substance Use Disorder}

To our knowledge, there have been no studies that focused on rTMS treatment for comorbid MDD and substance use disorder. However, HF-rTMS to the left DLPFC might be considered as possible treatment candidate; it has been reported that patients with cocaine use disorder showed a significant reduction in the Beck Depression Inventory (BDI) and the Symptom Checklist-90 Depression subscale scores after four weeks of left-sided HF-rTMS [47]. A systematic review and meta-analysis that included 26 RCTs concluded that HF-rTMS of the left DLPFC appears to be effective in reducing craving and substance consumption in patients with alcohol, nicotine, and illicit drug dependence [48]. The left DLPFC has been assumed to be involved in reward-based motivation [49], and hypoactive left DLPFC has been observed in those with substance dependence while they were performing cognitive tasks [50]. Because HF-rTMS of the left DLPFC has been considered as first-line therapy in patients with MDD, we believe this could be a potential treatment option for those with comorbid MDD and substance use disorder. Nevertheless, it would be best to avoid excitatory HF-rTMS when patients are withdrawing from alcohol use because they have a low seizure threshold.

\section{REQUIREMENT AND TIMEPOINT FOR CONSIDERING A CHANGE IN PROTOCOL}

When patients show limited improvement in symptoms, as determined using a structured evaluation tool (e.g., HAMD-21, BDI-II, etc.), after 10 sessions of standardized rTMS treatment, it is a time to consider a change in current protocol. Specifically, a retrospective study reported that a lack of clinical response (i.e., $<20 \%$ improvement in self-rated [BDI-II] scores) could predict poor response to rTMS treatment with $88 \%$ accuracy [51]. In addition, several patient- and illness-related factors, including absence of concomitant treatment with medication, long duration of current depressive episode, and presence of psychotic depression, older age, and high degree of treatment resistance, could be predictors of poor rTMS treatment responses (reviewed by Kar [52]). Furthermore, in a recent study evaluating the efficacy of iTBS, it showed that $20 \%$ improvement in the first week was the

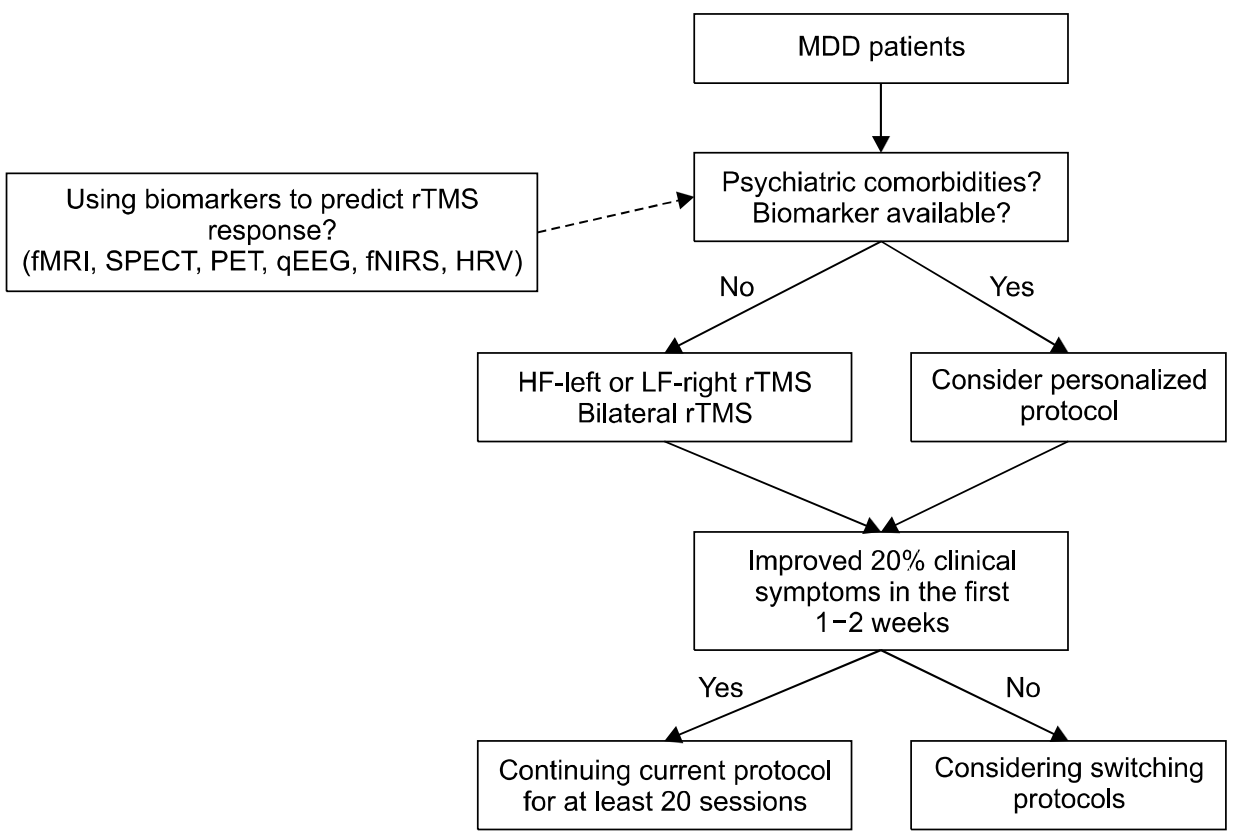

Fig. 1. Proposed paradigm for repetitive transcranial magnetic stimulation (rTMS) treatment in major depressive disorder (MDD) patients based on clinical conditions.

fMRI, functional magnetic resonance imaging; SPECT, single-photon emission computerized tomography; PET, positron emission tomography; qEEG, quantitative electroencephalography; fNIRS, functional near-infrared spectroscopy; HRV, heart rate variability; HF, high-frequency; LF, low-frequency. 
best predictor to the final antidepressant effects at second week [53]. Clinical experience suggests that 20 sessions should be conducted before declaring treatment failure and that some patients even need their treatment to be extended to 25-30 sessions for symptom improvements to occur [1]. However, non-responders, if identified early on, can be offered a switch of protocol, or an alternative treatment plan, which could improve the possibility of success.

\section{ALTERNATIVE PROTOCOLS WHEN PATIENTS SHOW NO RESPONSE TO UNILATERAL rTMS}

Firstly, in non-responders to HF-rTMS of the left DLPFC or LF-rTMS of the right DLPFC, shifting to LF-rTMS of the right DLPFC or HF-rTMS of the left DLPFC, respectively, is a straightforward decision (Fig. 1), which is recommended by the Canadian Network for Mood and Anxiety Treatments (CANMAT) [1]. Furthermore, the CANMAT recommends bilateral rTMS of the DLPFC (left HF and right LF in one session), which showed level 1 evidence, as the second-line treatment. Otherwise TBS could be considered as an alternative approach.

\section{Bilateral rTMS Using Low Frequency for the Right DLPFC Followed by High Frequency for the Left DLPFC}

It seems reasonable that sequential bilateral rTMS would be more effective than unilateral rTMS on the basis of the prefrontal asymmetry theory of MDD (i.e., hypoactivity of the left and hyperactivity of the right DLPFC, each presumably responsible for different symptom clusters) [9]. However, several previous studies (reviewed by Lefaucheur et al. [6]), except the one conducted by Blumberger et al. [54], have failed to demonstrate that the efficacy of bilateral stimulation is superior to that of unilateral stimulation, and hence, bilateral rTMS received a "probable efficacy” recommendation from a group of European experts [6]. In a study conducted by Blumberger et al., only bilateral rTMS (600 pulses at $1 \mathrm{~Hz}$ on the right DLPFC followed by 1,500 pulses at $10 \mathrm{~Hz}$ on the left DLPFC), but not unilateral HF-rTMS of the left DLPFC (2,100 pulses at $10 \mathrm{~Hz}$ ), produced significantly greater antidepressant effects than did the sham procedure in terms of remission or response (as measured using the HAMD-17 score). Two recently NMAs $[14,55]$ directly compared the efficacies among different protocols of rTMS and demonstrated that the efficacy of bilateral rTMS is superior to that of the sham procedure $(\mathrm{OR}, 3.69-4.92)$, even though the authors reported a lack of difference in the efficacies between bilateral rTMS and right-sided LF-rTMS or left-sided HF-rTMS. Therefore, the use of bilateral rTMS could be an alternative for the treatment of patients with MDD who did not show adequate response after 10 sessions of conventional unilateral rTMS protocol.

\section{TBS and Potential Mechanism of Its Effects on the Brain}

TBS, a novel rTMS pattern described by Huang et al. [56], is thought to induce more rapid and long-lasting effects on synaptic plasticity than are conventional rTMS protocols [57]. There are two TBS patterns that modulate cortical activity differentially. The cTBS is intended to reduce cortical excitability with the use of uninterrupted pulses, while the iTBS is administered by alternating the application of 10 bursts and non-stimulation intervals of 8 seconds [58]. The iTBS produces long-term potentiationlike effects, whereas cTBS produces a long-term depression-like cortical excitability reduction. The TBS protocols were proposed to offer the potential advantage of producing similar (if not larger) effects on cortical excitability and plasticity as do the conventional HF-/LF-rTMS protocols, but for a markedly shorter session duration (e.g., 3 minutes for an iTBS protocol vs. $>30$ minutes for a standard rTMS session) [16]. However, in a recent positron emission tomography (PET) study, Li et al. [59] found that TBS work by modulating fronto-cingulate circuit in TRD rather than directly affects the targeting DLPFC.

\section{Efficacy of TBS Against Depression}

Several randomized trials that targeted the left DLPFC with iTBS and biTBS (left iTBS and right cTBS), but not with right cTBS alone, have shown that their efficacy is superior to that of sham stimulation in patients with depression (Table 3) [6,60-64]. Specifically, a large, randomized, multicenter, non-inferiority trial $(N=414)$ was conducted in patients with MDD using a practical real-world approach [65]. Efficacy of three-minute iTBS sessions (120\% RMT, 600 pulses/session) was found to be non-inferior to that of the standard FDA-approved 37.5 minutes $10 \mathrm{~Hz}$ sessions in reducing depressive symptoms and self-reported measures of depression and in improving response and remission ( $49 \%$ and $32 \%$, respectively, for the 


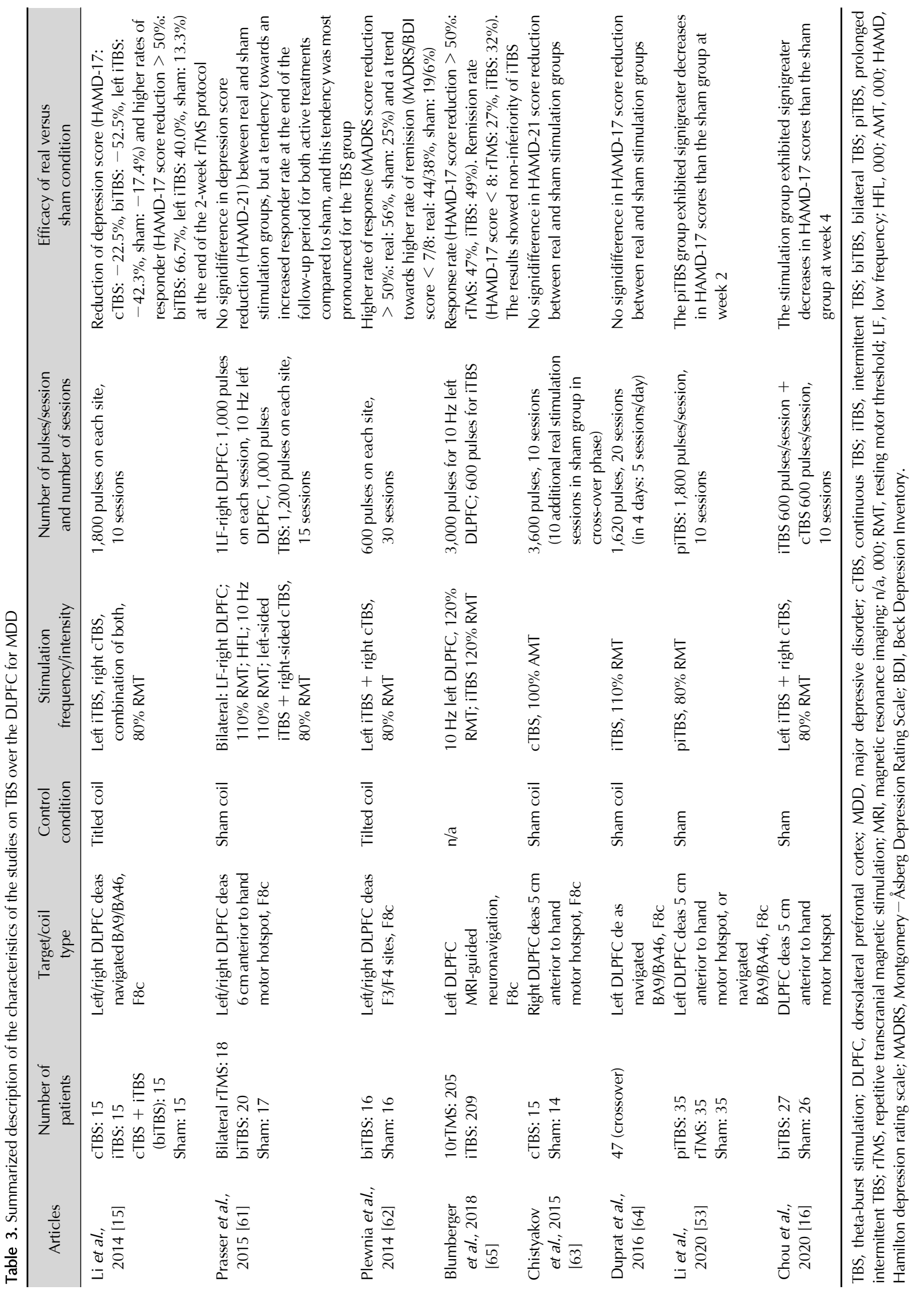


iTBS treatment group). iTBS administered using the parameters described in this trial was also approved for the treatment of MDD by the FDA. In addition, two recently published NMAs [14,55] directly compared efficacies among different modalities of rTMS/TBS and demonstrated that the efficacy of TBS (OR, 2.54), iTBS (OR, $3.20)$, or biTBS (OR, 4.44) is superior to that of sham treatment. Therefore, the use of TBS (either iTBS over the left DLPFC or biTBS over the bilateral DLPFC) could also be an alternative for the treatment of patients with MDD who did not show adequate response after 10 sessions of conventional HF-rTMS protocol. However, there has been few studies investigating the effects of TBS in MDD patients with other psychiatric comorbidities, even though recent data has indicated effectiveness of iTBS in reduce craving in substance-use disorders [66] and treating PTSD $[46,67]$.

\section{BIOMARKERS THAT PREDICT RESPONSE TO rTMS/TBS TREATMENT}

\section{Neuroimaging Correlates and Predictors of Treatment Responses}

PET and single-photon emission computerized tomography (SPECT) studies have found increased regional cerebral blood flow (rCBF) in the prefrontal and limbic regions following a course of rTMS [68,69]. First, Paillère Martinot et al. [70] found that at baseline, resting glucose metabolism on FDG-PET was lower in the left orbitofrontal cortex (OFC) and higher in the amygdala of rTMS non-responders than in those of responders. In addition, using a word generating task, Hernandez-Ribas et al. [71] found that small baseline functional magnetic resonance imaging ( $\mathrm{fMRI}$ ) deactivations in the right perigenual anterior cingulate cortex (ACC), left medial OFC, and left middle frontal gyrus were correlated with reduced depression severity following an HF-rTMS treatment, as were big activations in the left putamen. Furthermore, Baeken et al. $[72,73]$ found that higher levels of glucose metabolism in the DLPFC, ACC [72], and subgenual AAC (sgACC) [73] were related to better clinical response to both standard and accelerated HF-rTMS applied on the left DLPFC, respectively. Moreover, their study showed that clinical response corresponded to reduced activity in the sgACC following rTMS therapy [73], a finding indicating the central role of sgACC in response to depression treatment. On the contrary, LF stimulation to the right DLPFC has been found to elicit reduced rCBF in the right prefrontal cortex, left mediotemporal cortex, basal ganglia, and amygdala [69]. Superior responses to $1 \mathrm{~Hz}$ rTMS have been linked to the presence of a high $\mathrm{rCBF}$ at baseline in the left prefrontal regions, OFC, sgACC, insula, and limbic regions [74]. Furthermore, improvement in depressive symptoms was found to be related to a decrease in $\mathrm{rCBF}$ in the right prefrontal cortex, OFC, and right sgACC as well as the right putamen and insula [75].

In addition to regional activity markers, resting state functional connectivity of the sgACC has also been indicated as a potential correlate and predictor of rTMS treatment response. Specifically, Liston et al. [76] examined the relationship between rTMS treatment response and intra- or inter-neuro-network and found that hyperconnectivity of the sgACC with both default mode and central executive networks at baseline independently predicted better clinical outcomes. Moreover, Fox et al. [77] have suggested that a stronger negative connectivity between the DLPFC and sgACC at baseline may be related to better outcomes of rTMS treatment. These studies potentially implied the possibility of developing practical approaches for individualized rTMS targeting on the basis of sgACC connectivity [78]. Using a rostral ACC engagement task, Li et al. [79] reported that modulated prefrontal theta, which correlated with glucose metabolism in the rostral ACC, at baseline was able to predict antidepressant efficacy of $10 \mathrm{~Hz}$ rTMS to left DLPFC.

Functional near-infrared spectroscopy (fNIRS) is a recently developed functional neuroimaging technology that allows non-invasive measurements of the spatio-temporal characteristics of neural activity in frontotemporal regions [80]. While fNIRS has lower spatial resolution than does $\mathrm{fMRI}$, it has several advantages over the existing imaging techniques, including PET, SPECT, and fMRI [81], because it is non-invasive, easy to perform, tolerates small movements, is inexpensive, and provides excellent time resolution [82]. Moreover, fNIRS provides a bedside measurement of oxy-hemoglobin (oxy-Hb) and deoxy-hemoglobin (deoxy-Hb) concentrations, which are thought to indicate regional cerebral blood volumes and show strong correlations with fMRI signals [83]. Following this principle, fNIRS may be applicable to some of the unique research problems associated with neuropsychiatric disorders [84-86]. However, only few fNIRS studies focusing on its 
utility in predicting rTMS treatment response in patients with MDD have been published. Eschweiler et al. [87] found that absence of a task-related increase in total hemoglobin concentrations in the left DLPFC before the first active rTMS could significantly predict the clinical response to active rTMS. Furthermore, Shinba et al. [88] recorded the frontal hemoglobin concentration $(\mathrm{fHbC})$ during stimulation at the beginning and end of the TMS treatment using fNIRS and found that at the end of the stimulation, an increase in $\mathrm{fHbC}$ during stimulation was negatively correlated with the severity of depression and positively with an improvement in symptoms. Even though the results of using neuroimaging biomarkers to predict TMS treatment response is promising, several factors confound the accurate interpretation of findings across these studies, particularly in terms of variations in treatment parameters, imaging modalities, sample sizes, and analytical approaches. Replication and prospective examination of findings in future studies are warranted. At present, direct implementation of these predictive biomarkers is not clinically practical.

\section{EEG Correlates and Predictors of Outcome}

EEG is a graphic representation of the difference in voltage between two distinct brain points. Several EEG features or parameters have been studied intensively to predict patients with depression who may respond to TMS treatment. For instance, using the following resting state EEG (rsEEG) metrics, responders were distinguished from non-responders: higher (anterior) peak individual alpha frequency (IAF) values, lower power in the fronto-central theta frequency band, smaller P300 amplitudes in the parafacial zone $(\mathrm{Pz})$ during task, and increased prefrontal delta and beta cordance values [89]. However, in a later replication study, no significant differences between responders and non-responders were found in the IAF, frontal theta frequency band, or P300 amplitude [90]. In addition, some studies found that EEG asymmetry and background alpha $(8-13 \mathrm{~Hz})$ activity were not sufficient to predict response to HFL-rTMS [91], whereas other studies demonstrated a negative correlation between the background alpha power in the bilateral parieto-temporal regions and the change in the Beck Depression InventoryShort Form (BDI-SF) scores, after HF-rTMS of the left DLPFC [92]. Moreover, another study showed that an increase in low-theta band power in the sgACC at baseline predicted response to rTMS [93].

Cordance is a product of several algorithms involving absolute and relative EEG powers and has been shown to correlate well with cerebral perfusion and metabolism [94]. Two studies have found that an early change in quantitative EEG (QEEG) theta cordance could be a predictor of response to rTMS treatment administered to the prefrontal regions [95,96]. Moreover, Erguzel et al. [97] demonstrated that by applying the machine learning method based on artificial neural network, frontal slow band (delta and theta) cordance at baseline could be a predictor of response to rTMS with approximately $90 \%$ overall accuracy. Recently, several other novel EEG biomarkers such as permutation entropy or cumulative brain engagement index (CBEI) have been proposed. Permutation entropy is a non-linear measure that quantifies the complexity, or the irregularity, of a particular system and has been hypothesized to have higher predictive power for rTMS treatment response than have been other linear methods (such as frequency-based analysis) because of the complex non-linear characteristics of cerebral functions and neural processes [98]. Specifically, Shalbaf et al. [98] found that, within 7 days of initiating a course of rTMS ( $n=62$ ), treatment responders had a significantly higher entropy value than did non-responders, particularly in the prefrontal regions. In addition, a study by Isserles et al. [99] found significant group differences in two channel-derived cBEl after the first few rTMS treatment session between patients with depression who eventually responded and those who did not. Overall, while there are a number of promising EEG markers associated with positive rTMS treatment outcomes in patients with depression, currently there is insufficient data for the application of any of them in clinical practice. Results of a recent meta-analysis also underscore the current unavailability of sufficient evidence to recommend the use of EEG for guiding rTMS or other psychiatric treatment decisions at the present time [100].

\section{Heart Rate Variability as a Biomarker for Response to rTMS}

It is also well known that the autonomous nervous system in patients with MDD is often dysregulated, expressed as an overall high heart rate (HR) and low heart rate variability (HRV) $[101,102]$, which has been reported to be normalized after neuromodulation treatment [103]. 
Several studies have reported HR deceleration and HRV enhancement after the stimulation of the DLPFC using rTMS and transcranial direct current stimulation [104], which indicates that there is possibility for application of HRV as biomarkers to predict TMS treatment response. Several HRV parameters have been used in previous researches: within the frequency domain, absolute very low frequency power (VLF: $0.0033-0.04 \mathrm{~Hz}$ ), low frequency power (LF: $0.04-0.15 \mathrm{~Hz}$ ), and high frequency power ( $\mathrm{HF}: 0.15-0.4 \mathrm{~Hz}$ ) were calculated in $\mathrm{ms}^{2}$, as well as the ratio of low frequency power to high frequency power (LF/HF); the standard deviation of the NN (RR) intervals (SDNN) and root mean square of the successive differences (RMSSD) were taken within the time-domain. Currently, there are only two studies investigating this topic. Specifically, Udupa et al. [105] compared measures of cardiac autonomic function between patients with depression after two weeks of rTMS therapy and one month after selective serotonin reuptake inhibitor therapy (i.e., escitalopram $10 \mathrm{mg}$ per day), and they found that rTMS therapy is associated with improvements in HRV measures including SDNN, LF, HF, LF/HF in patients with depression. This improvement was only observed in those treated with rTMS but not with escitalopram. In another study by Iseger et al. [106], they found a trend toward an association between $\mathrm{HR}$ deceleration and treatment response, which explains $26 \%$ of the variance. Furthermore, several measures of HRV including LF, HF, SDNN, RMSSD were significantly higher during iTBS sessions than during sham sessions. Their data suggest that the larger the autonomic changes induced at baseline, the better the clinical response after 30 sessions of iTBS. In the future, more studies focusing on this topic are needed to confirm the usefulness of HRV parameters in predicting rTMS treatment responses.

\section{LIMITATIONS AND FUTURE DIRECTIONS}

Although the efficacies of rTMS and TBS for the treatment of depression have been proven by RCTs, few studies focused on psychotic depression, elderly, child, and adolescent populations, and patients comorbid with other psychiatric disorders such as anxiety disorder, PTSD, or substance abuse. In addition, none of the studies directly compared different rTMS treatment protocols in patients with MDD. Even though several NMAs provided the com- parative efficacy of different rTMS protocols and TBS, there are still some limitations we should be cautious about when interpreting their results [107]. Moreover, findings of studies that investigated biomarkers for the prediction of response to $\mathrm{rTMS} / \mathrm{TBS}$ in patients with MDD are not consistent or are scarce or limited by small sample sizes. Therefore, more studies focusing on this topic with larger numbers of patients are warranted.

\section{CONCLUSIONS}

Patients with MDD commonly have other psychiatric comorbidities, and personalized rTMS treatment plans are warranted to enhance the treatment responses. Switching rTMS protocols adequately may increase the possibility of treatment response. However, there are still limited numbers of TMS studies focusing on each specific psychiatric comorbidity in MDD patients, and more studies are needed to confirm the most optimal parameters for corresponding conditions. Moreover, understanding the biomarkers of patients with depression that could predict response to rTMS is important. However, currently there are still no reliable neurophysiological or neuroimaging biomarkers that could predict treatment response, which warrants future research.

\section{Acknowledgments}

We would like to than Dr. Pen-Tang Lin from Pen-Tang Psychiatric Clinic and Dr. Cheng-ta Li from Department of Psychiatry, Taipei Veterans General Hospital, Taiwan for their valuable comments in rTMS manuscript. The authors of this work were supported by the following grants: MOST 106-2314-B-039-027-MY3, 108-2320-B-039-048, 108-2813-C-039-133-B, 108-2314-B-039-016, 109-2320B-038-057-MY3, 109-2320-B-039-066, 109-2314-B-400-017, and 110-2321-B-006-004 from the Ministry of Science and Technology, Taiwan; NHRI-EX108-10528NI and NP109-PP-09 from the National Health Research Institutes, Taiwan; MYRG2018-00242-ICMS from University of Macau, China; ANHRF109-31 from An Nan Hospital, China Medical University, Tainan, Taiwan; CMRC-CMA-3 from Higher Education Sprout Project by the Ministry of Education (MOE), Taiwan; CMU108-SR-106 from the China Medical University, Taichung, Taiwan; and CMU104-S-16-01, CMU103-BC-4-1, CRS-108-048, DMR-108-216, DMR109-102, DMR-109-244, DMR-HHC-109-11 and DMR- 
HCC-109-12 from the China Medical University Hospital, Taichung, Taiwan.

\section{- Conflicts of Interest}

No potential conflict of interest relevant to this article was reported.

\section{Author Contributions}

Conceptualization: Po-Han Chou and Kuan-Pin Su. Supervision: Kuan-Pin Su. Writing - original draft: Po-Han Chou and Yen-Feng Lin. Writing-review \& editing: Ming-Kuei Lu, Hsin-An Chang, Che-Sheng Chu, Wei Hung Chang, Taishiro Kishimoto, Alexander T. Sack, and Kuan-Pin Su.

\section{ORCID}

\section{Po-Han Chou}

https://orcid.org/0000-0002-4148-457X

Yen-Feng Lin https://orcid.org/0000-0003-3017-6026

Ming-Kuei Lu https://orcid.org/0000-0002-2768-4399

Hsin-An Chang https://orcid.org/0000-0001-5572-7109

Che-Sheng Chu https://orcid.org/0000-0002-6911-1839

Wei Hung Chang https://orcid.org/0000-0002-5964-106X Taishiro Kishimoto https://orcid.org/0000-0003-0557-8648 Alexander T. Sack https://orcid.org/0000-0002-1471-0885 Kuan-Pin Su https://orcid.org/0000-0002-4501-2502

\section{REFERENCES}

1. Milev RV, Giacobbe P, Kennedy SH, Blumberger DM, Daskalakis ZJ, Downar J, et al. Canadian Network for Mood and Anxiety Treatments (CANMAT) 2016 clinical guidelines for the management of adults with major depressive disorder: Section 4. Neurostimulation treatments. Can J Psychiatry 2016;61:561-575.

2. Perera T, George MS, Grammer G, Janicak PG, PascualLeone A, Wirecki TS. The clinical TMS society consensus review and treatment recommendations for TMS therapy for major depressive disorder. Brain Stimul 2016;9:336-346.

3. Li CT, Su TP, Wang SJ, Tu PC, Hsieh JC. Prefrontal glucose metabolism in medication-resistant major depression. $\mathrm{Br} J$ Psychiatry 2015;206:316-323.

4. George MS, Lisanby SH, Avery D, McDonald WM, Durkalski $\checkmark$, Pavlicova M, et al. Daily left prefrontal transcranial magnetic stimulation therapy for major depressive disorder: a sham-controlled randomized trial. Arch Gen Psychiatry 2010;67:507-516.

5. O'Reardon JP, Solvason HB, Janicak PG, Sampson S, Isenberg $\mathrm{KE}, \mathrm{Nahas} Z$, et al. Efficacy and safety of transcranial magnetic stimulation in the acute treatment of major depression: a multisite randomized controlled trial. Biol Psychiatry 2007;62:1208-1216.

6. Lefaucheur JP, Aleman A, Baeken C, Benninger DH, Brunelin J, Di Lazzaro V, et al. Evidence-based guidelines on the therapeutic use of repetitive transcranial magnetic stimulation (rTMS): an update (2014-2018). Clin Neurophysiol 2020;131:474-528.

7. McClintock SM, Reti IM, Carpenter LL, McDonald WM, Dubin M, Taylor SF, et al. Consensus recommendations for the clinical application of repetitive transcranial magnetic stimulation (rTMS) in the treatment of depression. J Clin Psychiatry 2018;79:16cs10905.

8. Trevizol AP, Downar J, Vila-Rodriguez F, Thorpe KE, Daskalakis ZJ, Blumberger DM. Predictors of remission after repetitive transcranial magnetic stimulation for the treatment of major depressive disorder: an analysis from the randomised non-inferiority THREE-D trial. EClinicalMedicine 2020;22:100349.

9. Grimm S, Beck J, Schuepbach D, Hell D, Boesiger P, Bermpohl $\mathrm{F}$, et al. Imbalance between left and right dorsolateral prefrontal cortex in major depression is linked to negative emotional judgment: an $\mathrm{fMRI}$ study in severe major depressive disorder. Biol Psychiatry 2008;63:369-376.

10. Wassermann EM, Lisanby SH. Therapeutic application of repetitive transcranial magnetic stimulation: a review. Clin Neurophysiol 2001;112:1367-1377.

11. Chen J, Zhou C, Wu B, Wang Y, Li Q, Wei Y, et al. Left versus right repetitive transcranial magnetic stimulation in treating major depression: a meta-analysis of randomised controlled trials. Psychiatry Res 2013;210:1260-1264.

12. Lefaucheur JP, André-Obadia N, Antal A, Ayache SS, Baeken C, Benninger DH, et al. Evidence-based guidelines on the therapeutic use of repetitive transcranial magnetic stimulation (rTMS). Clin Neurophysiol 2014;125:2150-2206.

13. Fitzgerald PB, Hoy K, Gunewardene R, Slack C, Ibrahim S, Bailey $\mathrm{M}$, et al. A randomized trial of unilateral and bilateral prefrontal cortex transcranial magnetic stimulation in treatment-resistant major depression. Psychol Med 2011;41: 1187-1196.

14. Brunoni AR, Chaimani A, Moffa AH, Razza LB, Gattaz WF, Daskalakis ZJ, et al. Repetitive transcranial magnetic stimulation for the acute treatment of major depressive episodes: a systematic review with network meta-analysis. JAMA Psychiatry 2017;74:143-152.

15. Li CT, Chen MH, Juan $\mathrm{CH}$, Huang $\mathrm{HH}$, Chen LF, Hsieh JC, et al. Efficacy of prefrontal theta-burst stimulation in refractory depression: a randomized sham-controlled study. Brain 2014;137(Pt 7):2088-2098.

16. Chou PH, Lu MK, Tsai CH, Hsieh WT, Lai HC, Shityakov S, et al. Antidepressant efficacy and immune effects of bilateral theta burst stimulation monotherapy in major depression: a randomized, double-blind, sham-controlled study. Brain Behav Immun 2020;88:144-150. 
17. Cao X, Deng C, Su X, Guo Y. Response and remission rates following high-frequency vs. low-frequency repetitive transcranial magnetic stimulation (rTMS) over right DLPFC for treating major depressive disorder (MDD): a meta-analysis of randomized, double-blind trials. Front Psychiatry 2018;9: 413.

18. Feifel D. Network meta-analysis in mental health research. JAMA Psychiatry 2017;74:850-851.

19. Roth Y, Pell GS, Zangen A. Network meta-analysis in mental health research. JAMA Psychiatry 2017;74:851.

20. Berlim MT, Van den Eynde F, Daskalakis ZJ. Efficacy and acceptability of high frequency repetitive transcranial magnetic stimulation (rTMS) versus electroconvulsive therapy (ECT) for major depression: a systematic review and meta-analysis of randomized trials. Depress Anxiety 2013;30:614-623.

21. Murphy JA, Byrne GJ. Prevalence and correlates of the proposed DSM-5 diagnosis of Chronic Depressive Disorder. J Affect Disord 2012;139:172-180.

22. Rush AJ, Zimmerman M, Wisniewski SR, Fava M, Hollon SD, Warden D, et al. Comorbid psychiatric disorders in depressed outpatients: demographic and clinical features. J Affect Disord 2005;87:43-55.

23. Rodrigues PA, Zaninotto AL, Neville IS, Hayashi CY, Brunoni AR, Teixeira MJ, et al. Transcranial magnetic stimulation for the treatment of anxiety disorder. Neuropsychiatr Dis Treat 2019;15:2743-2761.

24. Balderston NL, Beydler EM, Roberts C, Deng ZD, Radman T, Lago T, et al. Mechanistic link between right prefrontal cortical activity and anxious arousal revealed using transcranial magnetic stimulation in healthy subjects. Neuropsychopharmacology 2020;45:694-702.

25. White D, Tavakoli S. Repetitive transcranial magnetic stimulation for treatment of major depressive disorder with comorbid generalized anxiety disorder. Ann Clin Psychiatry 2015;27:192-196.

26. Clarke E, Clarke P, Gill S, Paterson T, Hahn L, Galletly C. Efficacy of repetitive transcranial magnetic stimulation in the treatment of depression with comorbid anxiety disorders. J Affect Disord 2019;252:435-439.

27. Mantovani A, Aly M, Dagan Y, Allart A, Lisanby SH. Randomized sham controlled trial of repetitive transcranial magnetic stimulation to the dorsolateral prefrontal cortex for the treatment of panic disorder with comorbid major depression. J Affect Disord 2013;144:153-159.

28. Rao S, Zisook S. Anxious depression: clinical features and treatment. Curr Psychiatry Rep 2009;11:429-436.

29. Fava M, Rush AJ, Alpert JE, Balasubramani GK, Wisniewski $\mathrm{SR}$, Carmin CN, et al. Difference in treatment outcome in outpatients with anxious versus nonanxious depression: a STAR*D report. Am J Psychiatry 2008;165:342-351.

30. Diefenbach GJ, Bragdon L, Goethe JW. Treating anxious depression using repetitive transcranial magnetic stimulation. J Affect Disord 2013;151:365-368.
31. Chen L, Hudaib AR, Hoy KE, Fitzgerald PB. Is rTMS effective for anxiety symptoms in major depressive disorder? An efficacy analysis comparing left-sided high-frequency, right-sided low-frequency, and sequential bilateral rTMS protocols. Depress Anxiety 2019;36:723-731.

32. Kessler RC, Sonnega A, Bromet E, Hughes M, Nelson CB. Posttraumatic stress disorder in the National Comorbidity Survey. Arch Gen Psychiatry 1995;52:1048-1060.

33. Rytwinski NK, Scur MD, Feeny NC, Youngstrom EA. The co-occurrence of major depressive disorder among individuals with posttraumatic stress disorder: a meta-analysis. J Trauma Stress 2013;26:299-309.

34. Bernardy NC, Friedman MJ. Psychopharmacological strategies in the management of posttraumatic stress disorder (PTSD): what have we learned? Curr Psychiatry Rep 2015; 17:564.

35. Green BL, Krupnick JL, Chung J, Siddique J, Krause ED, Revicki D, et al. Impact of PTSD comorbidity on one-year outcomes in a depression trial. J Clin Psychol 2006;62: 815-835.

36. Post LM, Zoellner LA, Youngstrom E, Feeny NC. Understanding the relationship between co-occurring PTSD and MDD: symptom severity and affect. J Anxiety Disord 2011; 25:1123-1130.

37. Cirillo P, Gold AK, Nardi AE, Ornelas AC, Nierenberg AA, Camprodon J, et al. Transcranial magnetic stimulation in anxiety and trauma-related disorders: a systematic review and meta-analysis. Brain Behav 2019;9:e01284.

38. Hernandez MJ, Reljic T, Van Trees K, Phillips S, Hashimie J, Bajor L, et al. Impact of comorbid PTSD on outcome of repetitive transcranial magnetic stimulation (TMS) for veterans with depression. J Clin Psychiatry 2020;81:19m13152.

39. Rosenberg PB, Mehndiratta RB, Mehndiratta YP, Wamer A, Rosse RB, Balish M. Repetitive transcranial magnetic stimulation treatment of comorbid posttraumatic stress disorder and major depression. I Neuropsychiatry Clin Neurosci 2002; 14:270-276.

40. Osuch EA, Benson BE, Luckenbaugh DA, Geraci M, Post RM, McCann U. Repetitive TMS combined with exposure therapy for PTSD: a preliminary study. J Anxiety Disord 2009;23:54-59.

41. Philip NS, Ridout SJ, Albright SE, Sanchez G, Carpenter LL. 5-Hz transcranial magnetic stimulation for comorbid posttraumatic stress disorder and major depression. J Trauma Stress 2016;29:93-96.

42. Carpenter LL, Conelea C, Tyrka AR, Welch ES, Greenberg $\mathrm{BD}$, Price $\mathrm{LH}$, et al. $5 \mathrm{~Hz}$ repetitive transcranial magnetic stimulation for posttraumatic stress disorder comorbid with major depressive disorder. J Affect Disord 2018;235:414-420.

43. Cohen JE, Shalev H, Admon R, Hefetz S, Gasho CJ, Shachar $\mathrm{LJ}$, et al. Emotional brain rhythms and their impairment in post-traumatic patients. Hum Brain Mapp 2013;34:13441356. 
44. Boggio PS, Rocha M, Oliveira MO, Fecteau S, Cohen RB, Campanhã $\mathrm{C}$, et al. Noninvasive brain stimulation with high-frequency and low-intensity repetitive transcranial magnetic stimulation treatment for posttraumatic stress disorder. J Clin Psychiatry 2010;71:992-999.

45. Cohen H, Kaplan Z, Kotler M, Kouperman I, Moisa R, Grisaru N. Repetitive transcranial magnetic stimulation of the right dorsolateral prefrontal cortex in posttraumatic stress disorder: a double-blind, placebo-controlled study. Am J Psychiatry 2004; 161:515-524.

46. Philip NS, Barredo J, Aiken E, Larson V, Jones RN, Shea MT, et al. Theta-burst transcranial magnetic stimulation for posttraumatic stress disorder. Am J Psychiatry 2019;176:939948.

47. Pettorruso M, Martinotti G, Santacroce R, Montemitro C, Fanella F, di Giannantonio M, et al. rTMS reduces psychopathological burden and cocaine consumption in treatmentseeking subjects with cocaine use disorder: an open label, feasibility study. Front Psychiatry 2019;10:621.

48. Zhang JJQ, Fong KNK, Ouyang RG, Siu AMH, Kranz GS. Effects of repetitive transcranial magnetic stimulation (rTMS) on craving and substance consumption in patients with substance dependence: a systematic review and meta-analysis. Addiction 2019;114:2137-2149.

49. Balconi M, Finocchiaro R, Canavesio Y. Reward-system effect (BAS rating), left hemispheric "unbalance" (alpha band oscillations) and decisional impairments in drug addiction. Addict Behav 2014;39:1026-1032.

50. Eldreth DA, Matochik JA, Cadet JL, Bolla KI. Abnormal brain activity in prefrontal brain regions in abstinent marijuana users. Neuroimage 2004;23:914-920.

51. Feffer K, Lee HH, Mansouri F, Giacobbe P, Vila-Rodriguez F, Kennedy $\mathrm{SH}$, et al. Early symptom improvement at 10 sessions as a predictor of rTMS treatment outcome in major depression. Brain Stimul 2018;11:181-189.

52. Kar SK. Predictors of response to repetitive transcranial magnetic stimulation in depression: a review of recent updates. Clin Psychopharmacol Neurosci 2019;17:25-33.

53. Li CT, Cheng CM, Chen MH, Juan CH, Tu PC, Bai YM, et al. Antidepressant efficacy of prolonged intermittent theta burst stimulation monotherapy for recurrent depression and comparison of methods for coil positioning: a randomized, double-blind, sham-controlled study. Biol Psychiatry 2020;87: 443-450.

54. Blumberger DM, Maller JJ, Thomson L, Mulsant BH, Rajji TK, Maher M, et al. Unilateral and bilateral MRI-targeted repetitive transcranial magnetic stimulation for treatment-resistant depression: a randomized controlled study. J Psychiatry Neurosci 2016;41:E58-E66.

55. Mutz J, Vipulananthan V, Carter B, Hurlemann R, Fu CHY, Young AH. Comparative efficacy and acceptability of nonsurgical brain stimulation for the acute treatment of major depressive episodes in adults: systematic review and net- work meta-analysis. BMJ 2019;364:/1079.

56. Huang YZ, Edwards MJ, Rounis E, Bhatia KP, Rothwell JC. Theta burst stimulation of the human motor cortex. Neuron 2005;45:201-206.

57. Rachid F. Safety and efficacy of theta-burst stimulation in the treatment of psychiatric disorders: a review of the literature. J Nerv Ment Dis 2017;205:823-839.

58. Wischnewski M, Schutter DJ. Efficacy and time course of theta burst stimulation in healthy humans. Brain Stimul 2015;8:685-692.

59. Li CT, Chen MH, Juan CH, Liu RS, Lin WC, Bai YM, et al. Effects of prefrontal theta-burst stimulation on brain function in treatment-resistant depression: a randomized sham-controlled neuroimaging study. Brain Stimul 2018;11:10541062.

60. Trevizol AP, Blumberger DM. An update on repetitive transcranial magnetic stimulation for the treatment of major depressive disorder. Clin Pharmacol Ther 2019;106:747-762.

61. Prasser J, Schecklmann M, Poeppl TB, Frank E, Kreuzer PM, Hajak G, et al. Bilateral prefrontal rTMS and theta burst TMS as an add-on treatment for depression: a randomized placebo controlled trial. World J Biol Psychiatry 2015;16:57-65.

62. Plewnia C, Pasqualetti P, Grosse S, Schlipf S, Wasserka B, Zwissler B, et al. Treatment of major depression with bilateral theta burst stimulation: a randomized controlled pilot trial. J Affect Disord 2014;156:219-223.

63. Chistyakov AV, Kreinin B, Marmor S, Kaplan B, Khatib A, Darawsheh $\mathrm{N}$, et al. Preliminary assessment of the therapeutic efficacy of continuous theta-burst magnetic stimulation (CTBS) in major depression: a double-blind shamcontrolled study. J Affect Disord 2015;170:225-229.

64. Duprat R, Desmyter S, Rudi de R, van Heeringen K, Van den Abbeele $\mathrm{D}$, Tandt $\mathrm{H}$, et al. Accelerated intermittent theta burst stimulation treatment in medication-resistant major depression: a fast road to remission? J Affect Disord 2016; 200:6-14.

65. Blumberger DM, Vila-Rodriguez F, Thorpe KE, Feffer K, Noda Y, Giacobbe P, et al. Effectiveness of theta burst versus high-frequency repetitive transcranial magnetic stimulation in patients with depression (THREE-D): a randomised noninferiority trial. Lancet 2018;391:1683-1692.

66. Hanlon CA, Dowdle LT, Correia B, Mithoefer O, KearneyRamos T, Lench D, et al. Left frontal pole theta burst stimulation decreases orbitofrontal and insula activity in $\mathrm{CO}$ caine users and alcohol users. Drug Alcohol Depend 2017; 178:310-317.

67. Petrosino NJ, Wout-Frank MV, Aiken E, Swearingen HR, Barredo J, Zandvakili A, et al. One-year clinical outcomes following theta burst stimulation for post-traumatic stress disorder. Neuropsychopharmacology 2020;45:940-946.

68. Teneback CC, Nahas Z, Speer AM, Molloy M, Stallings LE, Spicer KM, et al. Changes in prefrontal cortex and paralimbic activity in depression following two weeks of daily 
left prefrontal TMS. J Neuropsychiatry Clin Neurosci 1999; 11:426-435.

69. Speer AM, Kimbrell TA, Wassermann EM, D Repella J, Willis $\mathrm{MW}$, Herscovitch $\mathrm{P}$, et al. Opposite effects of high and low frequency rTMS on regional brain activity in depressed patients. Biol Psychiatry 2000;48:1133-1141.

70. Paillère Martinot ML, Martinot JL, Ringuenet D, Galinowski A, Gallarda T, Bellivier F, et al. Baseline brain metabolism in resistant depression and response to transcranial magnetic stimulation. Neuropsychopharmacology 2011;36:2710-2719.

71. Hernández-Ribas R, Deus J, Pujol J, Segalàs C, Vallejo J, Menchón JM, et al. Identifying brain imaging correlates of clinical response to repetitive transcranial magnetic stimulation (rTMS) in major depression. Brain Stimul 2013;6:54-61.

72. Baeken C, De Raedt R, Van Hove C, Clerinx P, De Mey J, Bossuyt A. HF-rTMS treatment in medication-resistant melancholic depression: results from 18FDG-PET brain imaging. CNS Spectr 2009;14:439-448.

73. Baeken C, Marinazzo D, Everaert H, Wu GR, Van Hove C, Audenaert K, et al. The impact of accelerated HF-rTMS on the subgenual anterior cingulate cortex in refractory unipolar major depression: insights from 18FDG PET brain imaging. Brain Stimul 2015;8:808-815.

74. Kito S, Fujita K, Koga Y. Regional cerebral blood flow changes after low-frequency transcranial magnetic stimulation of the right dorsolateral prefrontal cortex in treatment-resistant depression. Neuropsychobiology 2008;58:29-36.

75. Kito S, Hasegawa T, Koga Y. Neuroanatomical correlates of therapeutic efficacy of low-frequency right prefrontal transcranial magnetic stimulation in treatment-resistant depression. Psychiatry Clin Neurosci 2011;65:175-182.

76. Liston C, Chen AC, Zebley BD, Drysdale AT, Gordon R, Leuchter B, et al. Default mode network mechanisms of transcranial magnetic stimulation in depression. Biol Psychiatry 2014;76:517-526.

77. Fox MD, Buckner RL, White MP, Greicius MD, PascualLeone A. Efficacy of transcranial magnetic stimulation targets for depression is related to intrinsic functional connectivity with the subgenual cingulate. Biol Psychiatry 2012;72:595-603.

78. Fox MD, Liu H, Pascual-Leone A. Identification of reproducible individualized targets for treatment of depression with TMS based on intrinsic connectivity. Neuroimage 2013;66: 151-160.

79. Li CT, Hsieh JC, Huang HH, Chen MH, Juan CH, Tu PC, et al. Cognition-modulated frontal activity in prediction and augmentation of antidepressant efficacy: a randomized controlled pilot study. Cereb Cortex 2016;26:202-210.

80. Chou PH, Huang CJ, Sun CW. The potential role of functional near-infrared spectroscopy as clinical biomarkers in schizophrenia. Curr Pharm Des 2020;26:201-217.

81. Chou PH, Lin WH, Li WR, Huang CM, Sun CW. Reduced language lateralization in first episode schizophrenia: a near infrared spectroscopy study. Prog Neuropsychopharmacol Biol Psychiatry 2017;78:96-104.

82. Chou PH, Tang KT, Chen YH, Sun CW, Huang CM, Chen DY. Reduced frontal activity during a verbal fluency test in fibromyalgia: a near-infrared spectroscopy study. J Clin Neurosci 2018;50:35-40.

83. Sato $\mathrm{H}$, Yahata $\mathrm{N}$, Funane $\mathrm{T}$, Takizawa R, Katura T, Atsumori $\mathrm{H}$, et al. A NIRS-fMRI investigation of prefrontal cortex activity during a working memory task. Neuroimage 2013;83:158173.

84. Chou PH, Lin WH, Hung CA, Chang CC, Li WR, Lan TH, et al. Perceived occupational stress is associated with decreased cortical activity of the prefrontal cortex: a multichannel near-infrared spectroscopy study. Sci Rep 2016;6: 39089.

85. Chou $\mathrm{PH}$, Lin WH, Lin CC, Hou PH, Li WR, Hung CC, et al. Duration of untreated psychosis and brain function during verbal fluency testing in first-episode schizophrenia: a near-infrared spectroscopy study. Sci Rep 2015;5:18069.

86. Chou PH, Koike S, Nishimura Y, Kawasaki S, Satomura Y, Kinoshita A, et al. Distinct effects of duration of untreated psychosis on brain cortical activities in different treatment phases of schizophrenia: a multi-channel near-infrared spectroscopy study. Prog Neuropsychopharmacol Biol Psychiatry 2014;49:63-69.

87. Eschweiler GW, Wegerer C, Schlotter W, Spandl C, Stevens A, Bartels $\mathrm{M}$, et al. Left prefrontal activation predicts therapeutic effects of repetitive transcranial magnetic stimulation (rTMS) in major depression. Psychiatry Res 2000;99: 161-172.

88. Shinba T, Kariya N, Matsuda S, Matsuda H, Obara Y. Increase of frontal cerebral blood volume during transcranial magnetic stimulation in depression is related to treatment effectiveness: a pilot study with near-infrared spectroscopy. Psychiatry Clin Neurosci 2018;72:602-610.

89. Arns M, Drinkenburg WH, Fitzgerald PB, Kenemans JL. Neurophysiological predictors of non-response to rTMS in depression. Brain Stimul 2012;5:569-576.

90. Krepel N, Sack AT, Kenemans JL, Fitzgerald PB, Drinkenburg $\mathrm{WH}$, Arns M. Non-replication of neurophysiological predictors of non-response to rTMS in depression and neurophysiological data-sharing proposal. Brain Stimul 2018;11: 639-641.

91. Price GW, Lee JW, Garvey C, Gibson N. Appraisal of sessional EEG features as a correlate of clinical changes in an rTMS treatment of depression. Clin EEG Neurosci 2008;39: 131-138.

92. Micoulaud-Franchi JA, Richieri R, Cermolacce M, Loundou A, Lancon C, Vion-Dury J. Parieto-temporal alpha EEG band power at baseline as a predictor of antidepressant treatment response with repetitive transcranial magnetic stimulation: a preliminary study. J Affect Disord 2012;137:156-160.

93. Narushima K, McCormick LM, Yamada T, Thatcher RW, 
Robinson RG. Subgenual cingulate theta activity predicts treatment response of repetitive transcranial magnetic stimulation in participants with vascular depression. J Neuropsychiatry Clin Neurosci 2010;22:75-84.

94. Leuchter AF, Cook IA, Lufkin RB, Dunkin J, Newton TF, Cummings JL, et al. Cordance: a new method for assessment of cerebral perfusion and metabolism using quantitative electroencephalography. Neuroimage 1994;1:208-219.

95. Bares M, Brunovsky M, Novak T, Kopecek M, Stopkova P, Sos $\mathrm{P}$, et al. QEEG theta cordance in the prediction of treatment outcome to prefrontal repetitive transcranial magnetic stimulation or venlafaxine $E R$ in patients with major depressive disorder. Clin EEG Neurosci 2015;46:73-80.

96. Hunter AM, Nghiem TX, Cook IA, Krantz DE, Minzenberg MJ, Leuchter AF. Change in quantitative EEG theta cordance as a potential predictor of repetitive transcranial magnetic stimulation clinical outcome in major depressive disorder. Clin EEG Neurosci 2018;49:306-315.

97. Erguzel TT, Ozekes S, Gultekin S, Tarhan N, Hizli Sayar G, Bayram A. Neural network based response prediction of rTMS in major depressive disorder using QEEG cordance. Psychiatry Investig 2015;12:61-65.

98. Shalbaf R, Brenner C, Pang C, Blumberger DM, Downar J, Daskalakis ZJ, et al. Non-linear entropy analysis in EEG to predict treatment response to repetitive transcranial magnetic stimulation in depression. Front Pharmacol 2018;9: 1188.

99. Isserles M, Daskalakis ZJ, George MS, Blumberger DM, Sackeim HA, Shahaf G. Simple electroencephalographic treatment-emergent marker can predict repetitive transcranial magnetic stimulation antidepressant response-a feasibility study. J ECT 2018;34:274-282.

100. Widge AS, Bilge MT, Montana R, Chang W, Rodriguez Cl,
Deckersbach T, et al. Electroencephalographic biomarkers for treatment response prediction in major depressive illness: a meta-analysis. Am J Psychiatry 2019;176:44-56.

101. Koenig J, Kemp AH, Beauchaine TP, Thayer JF, Kaess M. Depression and resting state heart rate variability in children and adolescents - a systematic review and meta-analysis. Clin Psychol Rev 2016;46:136-150.

102. Licht CM, de Geus EJ, Zitman FG, Hoogendijk WJ, van Dyck R, Penninx BW. Association between major depressive disorder and heart rate variability in the Netherlands Study of Depression and Anxiety (NESDA). Arch Gen Psychiatry 2008;65:1358-1367.

103. Kemp AH, Quintana DS, Gray MA, Felmingham KL, Brown K, Gatt JM. Impact of depression and antidepressant treatment on heart rate variability: a review and meta-analysis. Biol Psychiatry 2010;67:1067-1074.

104. Makovac E, Thayer JF, Ottaviani C. A meta-analysis of non-invasive brain stimulation and autonomic functioning: Implications for brain-heart pathways to cardiovascular disease. Neurosci Biobehav Rev 2017;74(Pt B):330-341.

105. Udupa K, Sathyaprabha TN, Thirthalli J, Kishore KR, Raju TR, Gangadhar BN. Modulation of cardiac autonomic functions in patients with major depression treated with repetitive transcranial magnetic stimulation. I Affect Disord 2007; 104:231-236

106. Iseger TA, Padberg F, Kenemans JL, Gevirtz R, Arns M. Neuro-Cardiac-Guided TMS (NCG-TMS): probing DLPFCsgACC-vagus nerve connectivity using heart rate - first results. Brain Stimul 2017;10:1006-1008.

107. Cipriani A, Higgins JP, Geddes JR, Salanti G. Conceptual and technical challenges in network meta-analysis. Ann Intern Med 2013;159:130-137. 